\title{
ERUPCIÓN DENTARIA: BASES MOLECULARES. UN ARTÍCULO DE REVISIÓN
}

\section{DENTAL ERUPTION: MOLECULAR BASES. A LITERATURE REVIEW}

Gabriela Silvia de la Cruz-Sedano ${ }^{*}$ (i) gadelacruz17@gmail.com

Abigail Katherine Ventura-Flores' ${ }^{10}$ abigail.katherine@hotmail.com

Jackeline Jajaira Jara-Porroa' ${ }^{10}$ jacky.jara.p@gmail.com

\section{Guido Alberto Perona-Miguel de Priego' guidoperona54@gmail.com}

Artículo recibido: 01/12/2019

Arbitrado por pares

Artículo aceptado: 24/03/2020

* Autor corresponsal:

Gabriela Silvia de la Cruz-Sedano gadelacruz17@gmail.com

\section{RESUMEN}

La erupción dental es un proceso dinámico que se inicia cuando se forma el germen dentario en su cripta de desarrollo hasta que el diente hace su aparición en boca. El folículo dental tiene un papel importante en la formación coronal y radicular del diente, y es esencial para la erupción dentaria. Para que un diente entre en erupción es necesario que exista resorción del hueso alveolar que cubre la corona del diente, de modo que se forme un camino a través del cual el diente se moverá. Para esto, se producen una serie de procesos moleculares y celulares localizados y programados genéticamente que permiten la osteogénesis y la osteoclastogénesis del hueso alveolar a fin de formar la vía de erupción. El objetivo de esta revisión es dar a conocer los posibles eventos celulares y moleculares que influyen en el proceso de erupción dentaria, ya que el mecanismo exacto aún es desconocido.

Palabras clave: erupción dentaria, bases moleculares

\section{ABSTRACT}

Dental eruption is a dynamic process, which begins when the dentary germ forms in the developmental crypt and finally appears in the mouth. The dental follicle has an important role in the coronal and root formation of the tooth and is essential for tooth eruption, without which the tooth could not erupt. Tooth eruption requires resorption of the alveolar bone that covers the crown of the tooth to form a path to the eruption and biological processes by which the tooth can move through this eruption path. Tooth eruption needs localized and genetically programmed molecular and cellular processes that allow osteogenesis and osteoclastogenesis of the alveolar bone to form the eruption path. The objective of this review was to describe the possible cellular and molecular events that influence the tooth eruption process, since the exact mechanism remains unknown.

Keywords: Dental eruption, molecular bases

\section{INTRODUCCIÓN}

Citar como: De la Cruz-Sedano G, VenturaFlores A, Jara-Porroa J, Perona-Miguel de Priego G. Erupción dentaria: bases moleculares. Un artículo de revisión. Rev Cient Odontol (Lima). 2020; 8(1): e009. DOI: $10.21142 / 2523-2754-0801-2020-009$
La erupción dental es un proceso dinámico que se inicia cuando se forma el germen dentario en su cripta de desarrollo hasta que el diente hace su aparición en boca. Este se mueve en dirección axial desde su posición original en el maxilar hasta su posición de oclusión $\left({ }^{1,2}\right)$.

\footnotetext{
1 División de Odontopediatría, Universidad Científica del Sur. Lima, Perú.
} 
Se ha demostrado que la corona del diente no es necesaria para la erupción dental; sin embargo, el folículo dental parece ser esencial para el proceso. La formación de una vía de erupción por la resorción ósea permite que la raíz se dirija hacia la cavidad oral, al mismo tiempo que se forma hueso en la base de la raíz ${ }^{3}$ ).

Los dientes derivan del ectodermo y el mesodermo, y la interacción entre las células epiteliales y las mesenquimatosas es vital para el inicio y la formación de los dientes. Las células ectodérmicas forman el órgano del esmalte y las células mesenquimatosas forman la papila dentaria que da origen a la dentina. También participan en la formación de los dientes las células de la cresta neural, que se integran con la papila dentaria, y las células epiteliales del órgano del esmalte inicial $(1,4)$.

La proliferación de las células ectodérmicas y el engrosamiento del ectodermo del estomodeo da lugar a la formación de la lámina dental, que se produce entre la cuarta y sexta semana de vida intrauterina. En ese momento, el epitelio ectodérmico está constituido por dos capas, una superficial y otra basal, conectadas al mesénquima por medio de la membrana basal. Las células basales del epitelio bucal inducidas por el ectomesénquima subyacente proliferan a todo lo largo del borde libre de los futuros maxilares, lo que da lugar a dos nuevas estructuras: la lámina vestibular y la lámina dentaria $\left({ }^{4}\right)$.

El folículo dental es una de las estructuras dentarias más importantes para el proceso de erupción, ya que tiene un papel fundamental en la formación de la corona y la raíz del diente, así como en la osteogénesis y la resorción ósea. El folículo dental proviene de la cresta neural del mesénquima, envuelve el órgano del esmalte y está destinado a convertirse en el ligamento periodontal. Es esencial para la erupción dentaria, pues sin él el diente no podría erupcionar $\left.{ }^{5-7}\right)$.

La formación de los dientes está bajo estricto control genético; se ha demostrado que más de 300 genes están asociados con su formación. Existe la hipótesis de que las células madre pueden estar presentes en el folículo dental y son capaces de diferenciarse en una amplia variedad de células. Recientes estudios a nivel molecular han tratado de evaluar las proteínas y genes marcadores relacionados con este proceso. El marcador GoPro49 es una proteína ubicada en el aparato de Golgi que induce al folículo en el proceso de la erupción. También se ha mostrado el marcador CD56 en el estadio de campana y el de papila $(1,5,8)$.

Tipos de erupción dentaria:

\section{Erupción activa}

Es el movimiento de los dientes desde que se forma en el hueso alveolar hasta llegar a su posición funcional en la cavidad oral $\left({ }^{9}\right)$.

\section{Movimiento preeruptivo}

Determinado por los gérmenes dentarios. Es el momento en el que los dientes se colocan dentro de la mandíbula para el movimiento eruptivo. La fase preeruptiva comienza tras el final de la etapa inicial de la campana hasta que el diente se ha formado completamente $\left({ }^{9,10}\right)$.

\section{Movimiento eruptivo}

Los movimientos de los dientes durante la fase eruptiva se subdividen en etapas intraóseas y etapas supraóseas. La fase eruptiva comienza con la formación de raíces y termina cuando el diente aparece en la cavidad bucal (fase prefuncional) $\left(^{9}\right)$.

\section{Movimiento poseruptivo}

Este movimiento mantiene la posición del diente en oclusión por compensación para el desgaste oclusal y proximal del diente. La fase comienza cuando los dientes alcanzan la oclusión y continúa mientras cada diente permanece en la cavidad oral (fase funcional) $\left(^{9}\right)$. 


\section{Erupción pasiva}

Es el resultado de los 3 movimientos de la erupción dental, caracterizado por el desplazamiento apical de la unión dentogingival. A medida que esto ocurre, la longitud de la corona clínica aumenta ya que el recubrimiento epitelial migra apicalmente, lo que da lugar a la corona clínica dentaria definitiva $\left({ }^{9}\right)$.

Para que un diente entre en erupción, se necesitan dos requisitos. Primero, tiene que existir resorción del hueso alveolar que cubre la corona del diente, de modo que forme un camino para la erupción. Segundo, tiene que haber un proceso biológico por el cual el diente se moverá a través de este camino de erupción $\left({ }^{4}\right)$.

\section{MOLÉCULAS IMPLICADAS EN LA ERUPCIÓN DENTARIA}

Cada diente hace erupción de forma independiente, por lo que las moléculas que inician la erupción, localización y regulación de los eventos celulares de erupción son diferentes para cada uno $\left(^{5}\right)$.

La determinación de las moléculas que requiere la erupción dental comenzó con el aislamiento de factor de crecimiento epidérmico (EGF) por Cohen en el año 1962, quien al inyectarla en roedores descubrió que se aceleraba la erupción del incisivo. Estudios posteriores han confirmado ampliamente estos resultados $y$ demostrado que, en las ratas, las inyecciones del factor de crecimiento epidérmico en el primer período posnatal de 0-3 días son críticos para maximizar los efectos de esta molécula $\left({ }^{5}\right)$.

Además, se observó que existe otro factor que aceleró la erupción de los incisivos en roedores y es el factor de crecimiento transformante alfa $(\mathrm{TGF}-\alpha)$. En vista de que ambas moléculas utilizan el mismo receptor, estas tendrían el mismo efecto sobre la erupción $\left(^{5}\right)$.

Por otro lado, se inyectó la molécula del factor de estimulación colonial (CSF-1) que induce la erupción dental en roedores sin dientes. La inyección de CSF-1 restauró la resorción ósea y la erupción dental. Es importante tener en cuenta que estas inyecciones se deben aplicar temprano tras el nacimiento para que ocurra la erupción, es decir, a más tardar el día 1, a fin de inducir la erupción del incisivo y no más tarde del día 3, para la primera erupción molar mandibular. La erupción molar en ratas normales también se acelera entre 3 y 4 días después de las inyecciones de CSF-1, un efecto que probablemente se deba a que este aumenta el número de células mononucleares positivas presentes en el folículo en el día 3, así como incrementa el número de osteoclastos en el hueso alveolar $\left(^{5}\right)$.

Un análisis más detallado de los factores de crecimiento epidérmico (EGF) y CSF-1 en la erupción de los dientes sugiere que pueden tener diferentes efectos sobre los incisivos respecto de la erupción molar. En un estudio de la comparación de sus efectos directos en la erupción, el EGF aceleró la erupción del incisivo, pero no de la molar, mientras que CSF-1 causó lo opuesto. Además, el EGF no aumentó el número de células mononucleares en el folículo dental de los molares ni el número de osteoclastos en el hueso alveolar alrededor de los molares, en contraste con los aumentos inducidos por CSF-1 ( $\left.{ }^{5}\right)$.

Entonces, el CSF-1 recluta las células mononucleares para la formación de osteoclastos que permite la reabsorción $\left({ }^{10-12}\right)$.

\section{REABSORCIÓN DEL HUESO ALVEOLAR}

Para entender los procesos moleculares y celulares que llevan a la erupción dental, se debe saber que el diente no erupcionado está revestido de hueso alveolar que debe ser reabsorbido para formar el camino del diente, es decir, la resorción ósea ensancha este canal para permitir que la corona del diente se mueva a través de él. Por lo tanto, la resorción ósea es un evento localizado y programado genéticamente, generado por los osteoclastos y que no requiere presión de los dientes al erupcionar $\left({ }^{1,5}\right)$.

Los cambios en el metabolismo del hueso son los que están asociados a la erupción dentaria y estos cambios dependen, a su vez, del folículo dentario $\left({ }^{13}\right)$. 


\section{PROCESOS MOLECULARES Y CELULARES EN EL FOLÍCULO DENTARIO QUE REGULAN LA REABSORCIÓN DE HUESO PARA LA ERUPCIÓN}

Los osteoclastos que se encuentran en el hueso alveolar son esenciales para la erupción dentaria. Las células mononucleares se fusionan, lo que permite la formación de los osteoclastos. Para el reclutamiento de células mononucleares se produce una cascada de señales paracrinas entre el retículo estrellado y el folículo dental. Dentro del folículo dental se da la síntesis y secreción de moléculas quimiotácticas $\mathrm{MCP}-1$ y CSF-1, que encargan de reclutar las células mononucleares que permiten la formación de osteoclastos. Estas moléculas (MCP-1 y CSF-1) interactúan con las expresiones genéticas mediante proteínas de TGF-b1, IL-1 $\alpha$ y PTHrP, que se encuentran en el retículo estrellado ( $\left.{ }^{5}\right)$ (fig. 1 ).

Para la formación de los osteoclastos se necesitan dos moléculas que se encuentran en el folículo dentario CSF-1 y el factor de diferenciación osteoclástica ODF, mientras que OPG inhibe este proceso al enviar señales entre las células $\left({ }^{5}\right)$.

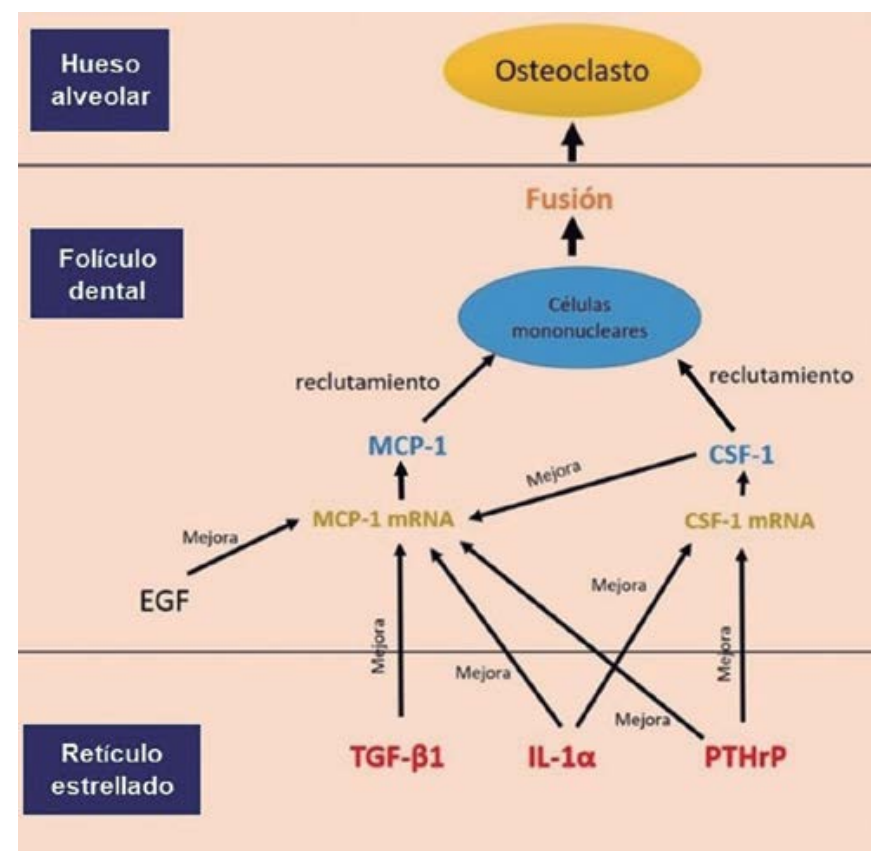

Figura I. Cascada de señales paracrinas entre el retículo estrellado y el folículo dental. Tomado y traducido de Wisse $\left({ }^{15}\right)$.

\section{PROCESOS MOLECULARES Y CELULARES EN EL FOLÍCULO DENTARIO QUE REGULAN LA FORMACIÓN DE HUESO PARA LA ERUPCIÓN}

Durante la fase intraósea de la erupción dentaria, es importante la formación del hueso alveolar en la base del diente. El hueso alveolar crece en la cresta del septum interradicular de las molares, lo que ayuda a su erupción. El folículo dental está implicado en el crecimiento de los huesos, pues resulta necesario para la erupción. Si se removiera el folículo dentario se inhibiría la erupción del diente y el crecimiento del hueso en la base de la cripta ósea $\left({ }^{15}\right)$.

La regulación de la osteogénesis tanto en forma cronológica como de espacio está regulada por genes específicos. La expresión del gen de BMP-2 comienza a aumentar el día 3 con una expresión máxima el día 9 después del nacimiento. Estos tiempos de expresión se correlacionan con el inicio de la formación de hueso alveolar en el día 3 y con su formación rápida en el día 9. De este modo, BMP-2 puede ser el gen regulador de la osteogénesis en la cripta ósea $\left({ }^{15}\right)$.

El ADN complementario (ADNc) codifica un factor de transcripción específico de osteoblastos 2 (Osf2). Este factor también se llama factor de unión central a1 (Cbfa1) o Runx2, el cual se encarga de la osteogénesis. Los factores de crecimiento con habilidades osteoinductoras conocidas como las BMP regulan la expresión de Cbfa1 para la diferenciación de osteoblastos durante la formación del hueso ${ }^{(5)}$.

A partir del folículo dentario se forma el ligamento periodontal que une al diente con el hueso alveolar al final de la fase intraósea. Durante la fase supraósea, el ligamento periodontal puede ayudar a levantar el diente hacia su plano oclusal ( $\left.{ }^{15}\right)$.

La presencia de células madre en el folículo dentario aumenta su potencial en el rol que tiene en la erupción del diente. Estas células, al ser pluripotenciales, son capaces de diferenciarse en condiciones apropiadas en otras células y tal vez ellas también contribuyan a la 
formación de algunos de los osteoclastos y osteoblastos necesarios para la erupción de los dientes $\left({ }^{15}\right)$.

En general, la erupción del diente es un evento localizado en el cual hay genes específicos que se encuentran en el folículo dentario que rodea el diente no erupcionado y que provocan la osteoclastogénesis y la osteogénesis necesarias para la erupción. Además de la regulación cronológica de estos genes, hay una localización espacial de algunos de ellos en el folículo dentario que ayudan a promover la resorción ósea alveolar en la porción coronal y la formación de hueso alveolar en la base del diente. Figura $2\left({ }^{15-16}\right)$.

El objetivo de esta revisión es dar a conocer los posibles eventos celulares y moleculares que influyen en el proceso de erupción dentaria, ya que el mecanismo exacto aún es desconocido y es importante conocer el tiempo y la secuencia de erupción, en la que podrían presentarse alteraciones que produzcan modificaciones $\left({ }^{17}\right)$.

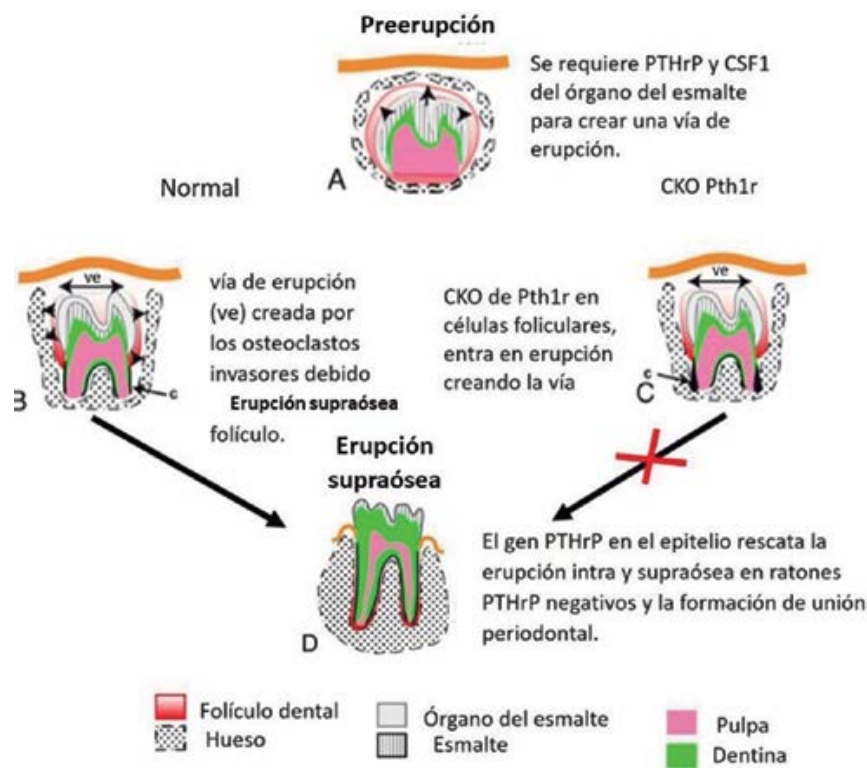

Figura 2. Fases de la erupción del diente y su regulación genética. Tomado y traducido de Richman $\left({ }^{16}\right)$.

(A) En la fase preeruptiva, los dientes están completamente rodeados por una cripta ósea. El órgano del esmalte produce PTHrP y CSFI (flechas) que retroalimentan el folículo dental. (B) La fase intraósea se caracteriza por la remoción espacialmente restringida de hueso en las señales coronarias del diente debido a las señales del folículo dental (flechas)". Los osteoclastos invaden y reabsorben el hueso para crear una vía de erupción (ve). El cemento comienza a formarse en las superficies de la raíz (c). (C) Los primeros molares crean una vía de erupción, pero no se mueven hacia el plano oclusal ( $X$ roja). Se forma un exceso de cemento (c), lo que quizás forma fusiones transitorias con el hueso y evita la erupción. Normalmente, los dientes se mueven a través de la vía de erupción (D) y se adhieren al hueso circundante a través del ligamento periodontal. Una eliminación completa de PTHrP, seguida de la restauración de la expresión en el órgano del esmalte utilizando KI4-Pthrp, rescata la erupción ( $\left.{ }^{16}\right)$.

\section{DISCUSIÓN}

La erupción de los dientes es un proceso complejo en el que se produce una serie de eventos moleculares y celulares que implica la acción oportuna y la interacción de las células del folículo dental, órgano dental, y el hueso alveolar.

Wise ${ }^{(5,15}$ ) señala que folículo dental parece jugar un papel esencial en el desencadenamiento de la remodelación del hueso del proceso alveolar que se requiere para la erupción de los dientes; sin embargo, otros numerosos factores influyen en el proceso de erupción, como los hormonales, sistémicos y morfológicos relacionados con el crecimiento y el desarrollo de la mandíbula.

Gaeta-Araujo et al. $\left({ }^{18}\right)$ analizaron el canal gubernacular (CG) y la fijación al folículo dentario para una erupción normal, y demostraron que, cuando el CG está unido al folículo dental en un sitio inusual, es probable que el diente tenga un proceso de erupción anormal.

Sandoval et al. $\left({ }^{19}\right)$ evaluaron la metaloproteinasa de matriz 2 (MMP-2), enzima proteolítica responsable de la degradación de la mayoría de los componentes de la matriz extracelular, y concluyeron que esta puede ser importante para la matriz extracelular durante el desarrollo dental y la secreción de sus tejidos mineralizados. También indican que la MMP-2 puede jugar un papel en la remodelación tisular extensa durante las fases intra y extraóseas del proceso de erupción de los dientes.

Kreiborg $\left({ }^{14}\right)$ indica que existen numerosas aberraciones genéticas que pueden afectar la formación y erupción de los dientes. La displasia cleidocraneal causada por mutaciones en el gen del factor de transcripción Runx2 produce alteraciones en la erupción dental. Estudios clínicos, modelos en animales y estudios de biología molecular han documentado que Runx2 es importante para la diferenciación de osteoblastos, para la regresión de la lámina dental y para la osteoclastogénesis en el folículo dental y el ligamento periodontal $\left(^{6}\right)$.

La falla primaria de erupción es una enfermedad rara con una prevalencia del $0,06 \%$. Se caracteriza por presentar 
una erupción dentaria incompleta a pesar de la presencia de una vía de erupción. Esta enfermedad está asociada con la mutación genética del gen PTH1R, esencial en el proceso de erupción del diente al causar la no irrupción parcial o completa de los dientes. La alteración de las células del folículo dental que producen PTHrP hace que el diente quede impactado o encapsulado en la cripta ósea $\left({ }^{11,12}\right)$.

Generalmente, los trastornos en la erupción están asociados con síndromes como el de GAPO. Existe poca literatura que explique la causa de una erupción prematura o tardía de los dientes. La erupción prenatal de los dientes es muy rara, pues se presenta en 1 de cada 2000 nacimientos $\left({ }^{20}\right)$.

La erupción de los dientes está mediada por numerosos factores que pueden provocar la anticipación o retraso

\section{REFERENCIAS BIBLIOGRÁFICAS}

1. Alzate F, Serrano L, Cortes L, Torres EA, Rodríguez MJ. Chronology and sequence of tooth eruption in the first transitional period. CES Odontol. 2016; 29 (1): 57-69.

2. Ayala Y, Carralero LC, Leyva BR. La erupción dentaria y sus factores influyentes. CCM. 2018; (4): 681-94.

3. Choukroune $\mathrm{C}$. Tooth eruption disorders associated with systemic and genetic diseases: clinical guide. J Dentofacial Anom Orthod. 2017; 20: 402.

4. Gómez M, Campos A. Histología y embriología bucodental. 2.a ed. Madrid: Panamericana; 2006.

5. Wise G, Frazier R, Souza D. Cellular, molecular, and genetic determinants of tooth eruption. Crit Rev Oral Biol Med. 2002; 13 (4): 323-34. doi: 10.1177/154411130201300403

6. Dorotheou D, Gkantidis N, Karamolegkou M, Kalyvas D, Kiliaridis S, Kitraki E. Tooth eruption: altered gene expression in the dental follicle of patients with cleidocranial dysplasia. Orthod Craniofac Res. 2013; 16: 20-7. doi: 10.1111/ocr.12000

7. Zhou T, Pan J, Wu P, et al. Dental follicle cells: roles in development and beyond. Stem Cells Int. 2019: 1-17.

8. Li J, Parada C, Chai Y. Cellular and molecular mechanisms of tooth root development. Development. 2017; 144 (3): 374-84.

9. Rabea A. Recent advances in understanding theories of eruption. Future Dental J. 2018; 4: 189-96.

10. Srinath SK, Sahana S, Vishwanath SK, Ritu S. Mechanism of tooth eruption \& its clinical significance - A systematic review of literature. Elixir Dentistry. 2013; 65: 19676-80. de la erupción dental, entre ellos los siguientes: sexo, raza, relación peso-talla, condición sistémica, entre otros. Por esto es necesario conocer los eventos celulares y moleculares involucrados en la erupción dentaria, lo que nos permitirá realizar acciones para evitar malas posiciones dentarias en el futuro.

Contribución de autoría: Gabriela Silvia de la CruzSedano, Abigail Katherine Ventura-Flores, Jackeline Jajaira Jara-Porroa y Guido Alberto Perona-Miguel de Priego han participado en la elaboración del artículo, la recolección de la información, la redacción y aprobación de la versión final.

Fuente de financiamiento: Autofinanciado.

Potenciales conflictos de interés: Los autores declaran no tener ningún tipo de conflicto de interés.
11. Hanisch M, Hanisch L, Kleinheinz J, Jung S. Primary failure of eruption (PFE): a systematic review. Head Face Med. 2018; 14 (1): 5. doi: 10.1186/s13005-018-0163-7

12. Jain U, Kallury A, Rao D, Bharti H. Primary failure of eruption (PFE). BMJ Case Rep. 2015. doi:10.1136/bcr-2015-209703

13. Gorski J, Marks S. Current concepts of the biology of tooth eruption. Crit Rev Oral Biol Med. 1992; 3 (3): 185-206.

14. Kreiborg S, Jensen BL. Tooth formation and eruption-lessons learnt from cleidocranial dysplasia. Eur J Oral Sci. 2018; 126 (1): 72-80.

15. Wise G. Cellular and molecular basis of tooth eruption. Orthod Craniofac Res. 2009; 12: 67-73. doi: 10.1111/j.16016343.2009.01439.x

16. Richman J. Shedding new light on the mysteries of tooth eruption. PNAS 2019; 116 (2): 353-5.

17. Sanabria A, Jiménez D, Gutiérrez T, Pilonieta G. Factores que influyen en la cronología de erupción de los dientes permanentes. Ustasalud Odontología 2006; 5: 132-6.

18. Gaeta H, Bronetti M, Tirapellic C, Queiroz D, Oliveira C. Detection of the gubernacular canal and its attachment to the dental follicle may indicate an abnormal eruption status. Angle Orthodontist, 2019; 89-5. doi: 10.2319/090518-651.1

19. Sandoval N, Lima N, Bautz W, Gama de Souza L, Coburn K. Matrix Metalloproteinase 2: a possible role in tooth development and eruption. Odovtos Int J Dental Sc. 2018; 21 (1): 41-51.

20. Kjær I. Mechanism of human tooth eruption: review article including a new theory for future studies on the eruption process. Scientifica 2014; 1-13. 